\title{
Biological correlates of day-to-day variation in flow-mediated dilation in individuals with Type 2 diabetes: a study of test-retest reliability
}

\author{
S. G. West ${ }^{1}$ P. Wagner ${ }^{1}$ S. L. Schoemer ${ }^{1}$ K. D. Hecker ${ }^{2}$ K. L. Hurston ${ }^{1}$ A. Likos Krick L. Boseska $^{1}$ • \\ J. Ulbrecht 1,3 - A. L. Hinderliter ${ }^{4}$ \\ ${ }^{1}$ Department of Biobehavioral Health, The Pennsylvania State University, University Park, USA \\ 2 Department of Nutritional Sciences, The Pennsylvania State University, University Park, Pennsylvania, USA \\ ${ }^{3}$ Department of Medicine, The Pennsylvania State University, Hershey, Pennsylvania, USA \\ ${ }^{4}$ Division of Cardiology, Department of Medicine, University of North Carolina at Chapel Hill, Chapel Hill, North Carolina, USA
}

\section{Abstract}

Aims/hypothesis. Dysfunction of the vascular endothelium is commonly observed in Type 2 diabetes, and endothelial function may be an important outcome for clinical trials in diabetic samples. However, the most commonly used non-invasive test of endothelial function (flow-mediated dilation [FMD]) is technically challenging to perform, and no previous studies have carefully examined the reproducibility of FMD measurements in individuals with Type 2 diabetes. In this study, we tested the hypothesis that larger day-to-day changes in insulin and glucose are associated with larger fluctuations in FMD.

Methods. Ultrasound was used to measure the FMD (\% change from baseline diameter) of the brachial artery in 18 healthy adults with Type 2 diabetes on three separate occasions, in the absence of changes to diet, activity level or medications. The CV and mean deviations between pairs of FMD scores in the same individual were used as the primary outcome variables.
Results. The CV for FMD (29.7\%) was higher than the level traditionally accepted for biochemical assays. However, this CV estimate is within the low range of published values for FMD in healthy individuals. FMD scores were not significantly correlated with glucose or insulin levels. However, subjects with the largest variability in FMD also showed the largest fluctuations in glucose $(r=0.52)$, insulin $(r=0.47)$ and heart rate $(r=0.48)(p \leq 0.05)$.

Conclusions/interpretation. FMD can be reliably measured in individuals with Type 2 diabetes, and population-specific data on reliability is critical for the design of adequately powered studies of endothelial function.

Keywords Coefficient of variation .

Endothelium-dependent vasodilation .

Flow-mediated dilation - Glucose $\cdot$ Heart rate .

Insulin resistance $\cdot$ Test-retest reliability ·

Type 2 diabetes mellitus
Received: 17 March 2004 / Accepted: 4 May 2004

Published online: 9 September 2004

(C) Springer-Verlag 2004

S. G. West $(\bullet)$

Department of Biobehavioral Health,

The Pennsylvania State University,

315 Health and Human Development Building East,

University Park, PA 16802, USA

E-mail: sgw2@psu.edu

Tel.: +1-814-8630176, Fax: +1-814-8637525

Abbreviations: FMD, flow-mediated dilation .

$\mathrm{HOMA}_{\mathrm{IR}}$, homeostasis model assessment of insulin resistance ·

QUICKI, quantitative insulin sensitivity check index

\section{Introduction}

Dysfunction of the vascular endothelium is considered to be a precursor of atherosclerosis [1], and Type 2 diabetes is associated with both endothelial dysfunction [2] and increased risk of cardiovascular disease [3]. However, dietary [4] and pharmacological [5] interventions significantly improve endothelial function, even in this high-risk group. One non-invasive technique for assessing endothelial health uses ultrasound to examine the magnitude of the increase in diameter in the brachial artery after an increase in blood flow $[6,7]$, a phenomenon called flow-mediated dilation (FMD) [8]. Impaired FMD may be an independent 
Table 1. Studies reporting the CV for FMD and artery diameters in healthy adults

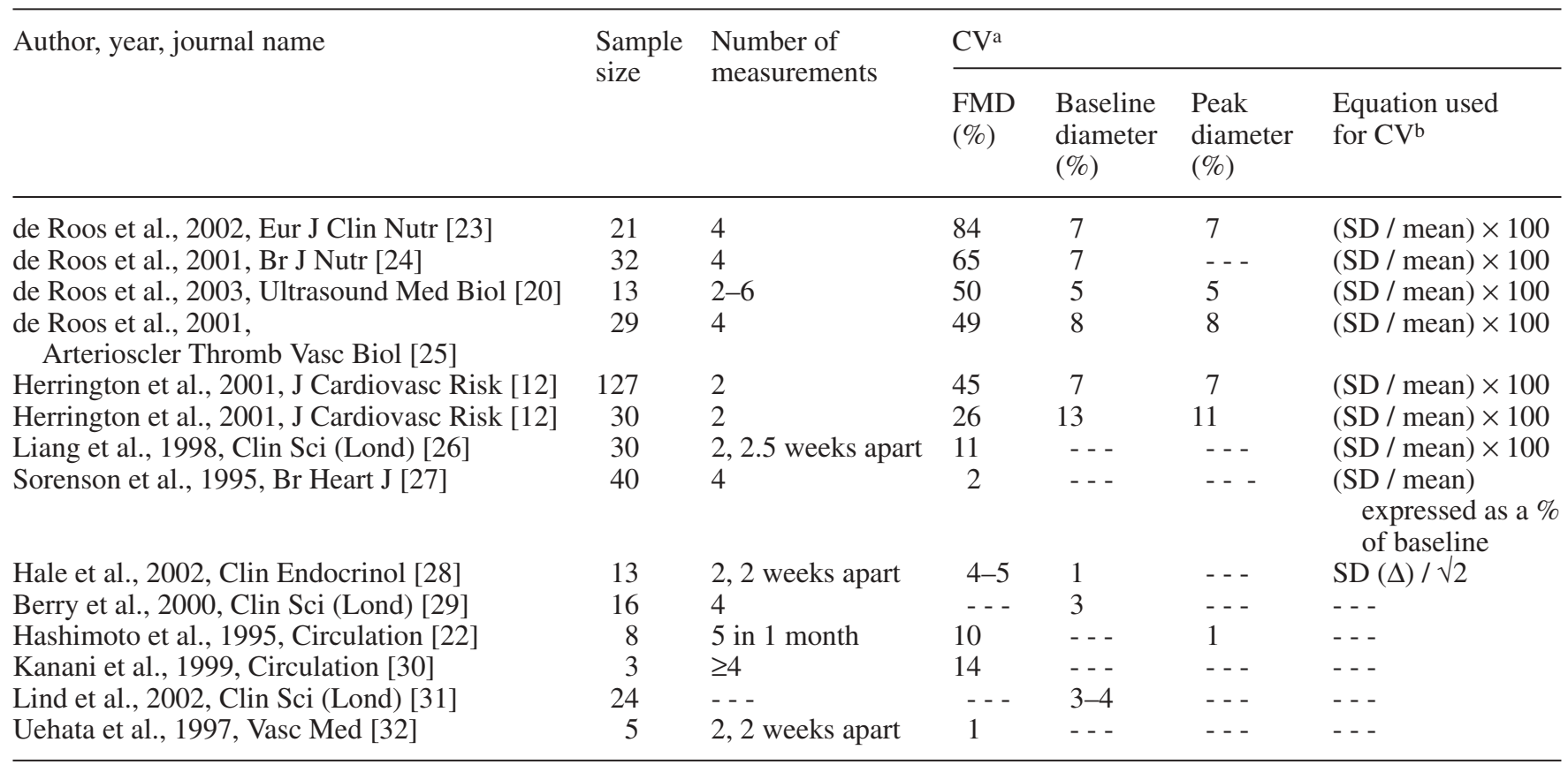

Dashed line (- -) indicates that information was not reported in the manuscript. ${ }^{\text {a }}$ Results were rounded to allow comparison across studies; $b$ if reported in the text

predictor of coronary events [9], and FMD scores are inversely correlated with a number of coronary risk markers [6], including insulin resistance [10], high WHR [11] and dyslipidaemia [6].

FMD can be measured using ultrasound equipment found in most medical centres and involves little discomfort or risk for patients. However, given the small diameter of the brachial artery $(2-5 \mathrm{~mm})$ and the large number of extraneous variables that can affect FMD $[12,13]$, it is challenging to measure it reliably. Published estimates of within-subject variability in FMD differ substantially (Table 1), and no studies to date have measured reliability in patients with diabetes. Variability is often attributed to sonographer technique and measurement error; however, it is also possible that day-to-day variations are caused by fluctuations in biological parameters such as glucose, insulin and triglycerides. In the present study, we examined the stability of FMD estimates in healthy adults with diabetes and examined the implications of any variability for future intervention studies in this population.

\section{Subjects and methods}

Subjects. We recruited 18 adults (aged 41-73 years) with Type 2 diabetes for a study of the acute effects of unsaturated fatty acids on vascular reactivity [14]. Patients were not insulin dependent and were otherwise reasonably healthy. Only the fasting data from the study are reported here. The sample included two postmenopausal women, one premenopausal woman and 15 men. Because hormone fluctuations across the menstrual cycle affect endothelial function, the premenopausal woman was examined during the early follicular phase of three consecutive menstrual cycles and women using oral contraceptives or hormone replacement therapy were excluded. Table 2 shows the cardiovascular risk characteristics of the subjects. The average BMI of the subjects in this sample was $29.3 \mathrm{~kg} / \mathrm{m}^{2}$.

Study design. Each participant was tested after a 12-h fast on three occasions, separated by at least 1 week (with the exception of the premenopausal female participant, all three tests were completed within 60 days). Participants were asked to avoid alcohol for $48 \mathrm{~h}$, to discontinue medications the night before each test and to maintain similar exercise and diet habits throughout the study. The study was approved by the Office of Regulatory Compliance at the Pennsylvania State University and written informed consent was obtained from each participant.

Ultrasound assessments. An Acuson 128XP duplex ultrasound imaging system (Siemens Medical Solutions, Malvern, Pa., USA) with a $10-\mathrm{MHz}$ linear array transducer was used to measure brachial artery diameter at baseline during reactive hyperaemia according to published guidelines [7]. Ischaemia was induced by inflating a BP cuff on the forearm (distal to the target artery) to $50 \mathrm{~mm} \mathrm{Hg}$ above systolic BP using an automated device (D. E. Hokanson, Bellevue, Wash., USA). Continuous, longitudinal, two-dimensional images of the brachial artery at 5 to $10 \mathrm{~cm}$ above the elbow of the right arm were obtained and stored on SVHS tape during quiet rest (1 min), cuff occlusion ( $5 \mathrm{~min}$ ) and reactive hyperaemia $(2 \mathrm{~min})$. The ultrasound examinations were performed by a single registered vascular technologist (P. Wagner). Flow velocity was measured using duplex pulsed Doppler with the ultrasound beam at two time points: at the beginning of baseline and immediately after cuff release. Flow ( $\mathrm{ml} / \mathrm{min})$ was calculated using the following equation: velocity time integral $\times$ cross-sectional area of the vessel $\left(\pi r^{2}\right) \times$ heart rate. 
Table 2. Vascular and metabolic parameters measured under fasting conditions across the three testing sessions

\begin{tabular}{|c|c|c|c|c|}
\hline & Visit one & Visit two & Visit three & Average CV $(\%)^{\mathrm{a}}$ \\
\hline FMD (\% change) & $5.57 \pm 0.54$ & $5.72 \pm 0.54$ & $5.51 \pm 0.54$ & 29.7 \\
\hline $\mathrm{CV}^{\prime}$ for FMD $(\%)^{\mathrm{b}}$ & --- & -- & --- & 1.2 \\
\hline \multicolumn{5}{|l|}{ Resting values } \\
\hline Blood flow (ml/min) & $105.4 \pm 6.6$ & $98.8 \pm 6.6$ & $101.8 \pm 6.6$ & 17.7 \\
\hline Blood velocity $(\mathrm{cm} / \mathrm{s})$ & $0.968 \pm 0.054$ & $0.932 \pm 0.053$ & $0.965 \pm 0.053$ & 10.8 \\
\hline \multicolumn{5}{|l|}{ Peak (hyperaemic) response } \\
\hline Blood flow (ml/min) & $709.2 \pm 52.4$ & $673.9 \pm 52.7$ & $647.05 \pm 52.6$ & 21.9 \\
\hline Blood velocity $(\mathrm{cm} / \mathrm{s})^{\mathrm{d}}$ & $1.78 \pm 0.09$ & $1.66 \pm 0.09$ & $1.60 \pm 0.09^{c}$ & 16.6 \\
\hline Artery diameter (mm) & $4.23 \pm 0.14$ & $4.23 \pm 0.14$ & $4.25 \pm 0.14$ & 2.5 \\
\hline Systolic BP (mm Hg) & $120.7 \pm 2.4$ & $119.6 \pm 2.4$ & $119.9 \pm 2.4$ & 4.2 \\
\hline Diastolic BP $(\mathrm{mm} \mathrm{Hg})^{\mathrm{d}}$ & $68.1 \pm 1.1$ & $68.3 \pm 1.1$ & $66.7 \pm 1.1$ & 4.2 \\
\hline Total cholesterol $(\mathrm{mmol} / \mathrm{l})$ & $195.2 \pm 5.0$ & $191.8 \pm 5.0$ & $190.1 \pm 5.0$ & 6.2 \\
\hline Triglycerides $(\mathrm{mmol} / \mathrm{l})^{\mathrm{d}}$ & $208.4 \pm 15.7$ & $174.0 \pm 15.7^{\mathrm{c}}$ & $182.0 \pm 5.7 \mathrm{c}$ & 18.0 \\
\hline HDL cholesterol (mmol/l) & $42.8 \pm 1.9$ & $43.1 \pm 1.9$ & $42.7 \pm 1.9$ & 6.6 \\
\hline LDL cholesterol (mmol/l) & $111.9 \pm 5.1$ & $113.9 \pm 5.0$ & $111.0 \pm 5.0$ & 9.1 \\
\hline Glucose $(\mathrm{mmol} / \mathrm{l})^{\mathrm{d}}$ & $6.87 \pm 0.36$ & $7.04 \pm 0.36$ & $7.24 \pm 0.36^{\mathrm{c}}$ & 7.8 \\
\hline Insulin $(\mathrm{pmol} / \mathrm{l})$ & $107.0 \pm 11.8$ & $115.3 \pm 11.8$ & $112.5 \pm 11.8$ & 22.8 \\
\hline QUICKI & $0.13 \pm 0.00$ & $0.13 \pm 0.00$ & $0.13 \pm 0.00$ & 3.0 \\
\hline $\mathrm{HOMA}_{\mathrm{IR}}$ & $5.14 \pm 0.60$ & $5.62 \pm 0.60$ & $5.42 \pm 0.60$ & 22.5 \\
\hline
\end{tabular}

Values are means \pm SEM unless otherwise indicated. All measures were collected after a $12-\mathrm{h}$ fast. ${ }^{a} \mathrm{CV}=(100 \times \mathrm{SD}) / \mathrm{mean}$; ${ }^{\mathrm{b}} \mathrm{CV}^{\prime}=(100 \times \mathrm{SD}) /($ mean +100$) ;{ }^{\mathrm{c}} p \leq 0.04$ vs visit one using the Tukey post hoc test; ${ }^{\mathrm{d}}$ main effect of time, $p \leq 0.04$

Analysis of arterial diameters. Images for analysis were sampled at end diastole using Brachial Imager software (Medical Imaging Applications, Iowa City, Iowa, USA). This yielded approximately 50 to 60 frames at baseline and 100 to 140 frames during the post-deflation sequence. Within each frame, diameters were repeatedly measured along a segment of vessel 2 to $8 \mathrm{~mm}$ long using automated edge-detection software (Brachial Analyzer; MIA, Iowa City, Iowa, USA) [15]. Each sequence of ultrasound images was reviewed by a single technician and scores were confirmed by a second observer (S. L. Schoemer). If FMD estimates differed by more than $1.5 \%$, consensus was reached by a third scorer (S. G. West). The same arterial landmark [16] was used for all scans collected from a single patient.

The average value of all interpretable images over the 1-min baseline period was used for resting arterial diameter. Peak FMD (FMD $\left.{ }_{\text {peak }}\right)$ was calculated by identifying the postdeflation image with the largest average diameter and using this value to calculate percentage change from baseline. We examined whether increasing the number of frames used to estimate the peak diameter would produce more consistent estimates. $\mathrm{FMD}_{\text {peak } \pm 5}$ was calculated using the average of the peak diameter, the five frames preceding the peak and the five frames following the peak. Similar calculations were performed using the average of two, four, six or eight additional frames surrounding the peak. Unless otherwise specified, $\mathrm{FMD}_{\text {peak } \pm 5}$ was reported here.

Lipids, lipoproteins, glucose and insulin were measured using conventional methods as described previously [14]. The homeostasis model assessment for insulin resistance $\left(\mathrm{HOMA}_{\mathrm{IR}}\right)$ was calculated using the following formula: fasting plasma glucose $(\mathrm{mmol} / \mathrm{l}) \times$ fasting insulin $(\mu \mathrm{U} / \mathrm{ml}) / 22.5$ [17]
We also calculated the quantitative insulin sensitivity check index (QUICKI), a recently proposed indicator of insulin sensitivity using the following equation: $1 /$ (log insulin $\times \log$ glucose), where glucose is measured in $\mathrm{mg} / \mathrm{dl}$ and insulin is measured in $\mu \mathrm{U} / \mathrm{ml}[18]$.

An oscillometric monitor (Dinamap Pro 100, Critikon, Tampa, Fla., USA) was used to measure BP. Stroke volume, cardiac output and total peripheral vascular resistance were estimated every other minute during resting periods via impedance cardiography as described previously [19].

Statistical analyses. We conducted a series of repeated-measures ANOVA using mixed models (SAS Version 8.2, SAS Institute, Cary, N.C., USA) to examine within-subject change in the outcome variables over repeated visits. Pearson correlations, adjusting for basal artery diameter, were used to test whether average fasting FMD was correlated with any of the demographic or cardiac-risk parameters.

The CV was calculated for each subject from the mean and SD for the three measurements as follows: $\mathrm{CV}=$ $(100 \times \mathrm{SD}) /$ mean. Herrington et al. [12] noted that many published studies have used an alternative method of calculating CV, which they termed CV' : CV' $=(100 \times \mathrm{SD}) / 100+$ mean. The CV was calculated for all variables, whereas CV' was only reported for FMD. Additional variability measures included: (i) correlations across successive visits; and (ii) mean variability (the difference between pairs of measurements, expressed as the absolute value, averaged across all three pairs of measurements from an individual). We examined whether the mean variability in FMD was correlated with the mean variability in haemodynamic measures (blood flow, BP, heart rate and total peripheral vascular resistance) or with variations in 
Table 3. Test-retest reliability of FMD-related variables

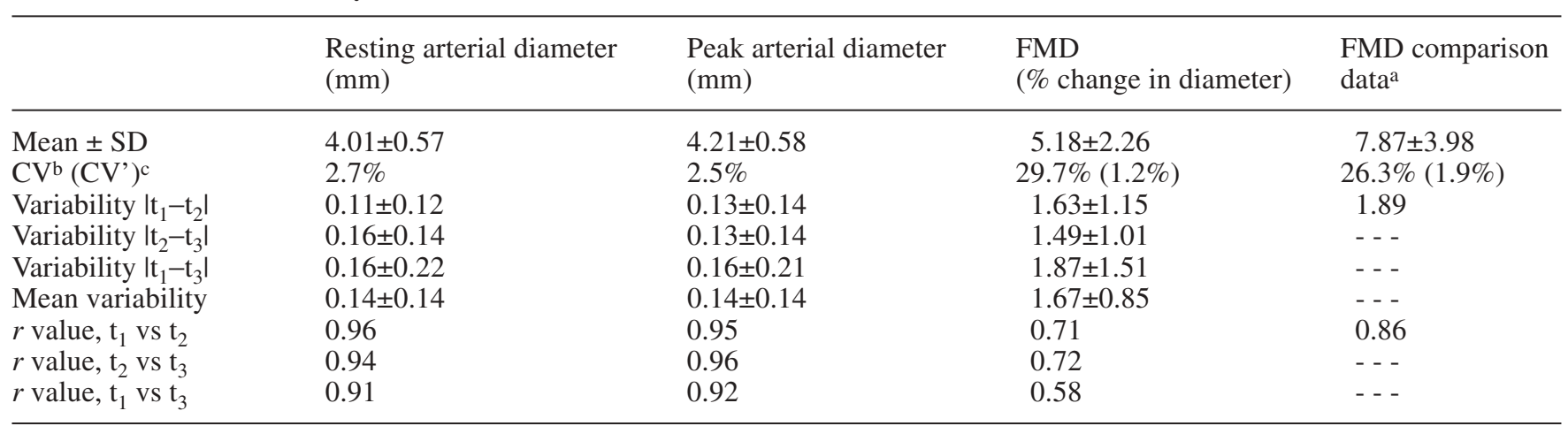

a FMD comparison data are taken from a paper by Herrington et al. [12] who measured FMD twice in healthy adults using a similar protocol; ${ }^{\mathrm{b}} \mathrm{CV}=(100 \times \mathrm{SD}) /$ mean; ${ }^{\mathrm{b}} \mathrm{CV}^{\prime}=(100 \times \mathrm{SD}) /($ mean +100$)$

glucose, insulin or lipids/lipoproteins. Multivariable regression analysis was used to estimate how much of the variance in FMD over the three visits could be explained by the biological predictors. A $p$ value less than or equal to 0.05 was considered statistically significant.

Finally, we estimated the sample sizes for crossover and parallel-arm designs using two-sided tests, power $=0.80-0.90$ and alpha $=0.05$. The within-subject SD was estimated using the equation $\mathrm{SD}_{\text {within }}=\sqrt{\mathrm{MSE}}$ (where the MSE is the mean square error from ANOVA with subject as a main effect) [20].

\section{Results}

There were few systematic changes in the outcome variables across the three measurements (Table 2). There were no significant changes in FMD or hyperaemic-flow volume across the three visits. Diastolic $\mathrm{BP}(-1.4 \mathrm{~mm} \mathrm{Hg})$, triglycerides $(-16 \mathrm{mmol} / \mathrm{l})$ and hyperaemic-flow velocity $(-22 \%)$ decreased significantly $(p \leq 0.02)$ from the first to the third visit. In contrast, fasting glucose was $0.39 \mathrm{mmol} / \mathrm{l}$ higher at the third visit $(p=0.04)$.

Predictors of fasting FMD. Lower FMD was associated with increasing age $(r=-0.72, p=0.001)$ and higher systolic BP $(r=-0.50, p=0.04)$. Subjects with high fasting triglycerides $(\geq 1.69 \mathrm{mmol} / \mathrm{l})$ had lower FMD scores than subjects with low or normal fasting levels (mean FMD $=3.76 \pm 0.74$ vs $6.31 \pm 0.66$, $p=0.03$ ). None of the other demographic, metabolic or cardiovascular-risk variables were significant predictors of fasting FMD, and fasting glucose was not related to fasting FMD in individuals either across visits or in the sample as a whole (Fig. 1).

Reliability of vascular ultrasound measures. Reliability statistics for FMD, artery diameter, arterial-bloodflow volume and blood-flow velocity are shown in Tables 3 and 4. The CV for FMD was $29.7 \%$, while the mean value for CV' was $1.2 \%$. The absolute diameters at baseline and peak dilation were measured more reliably than FMD itself was, and FMD variabil-

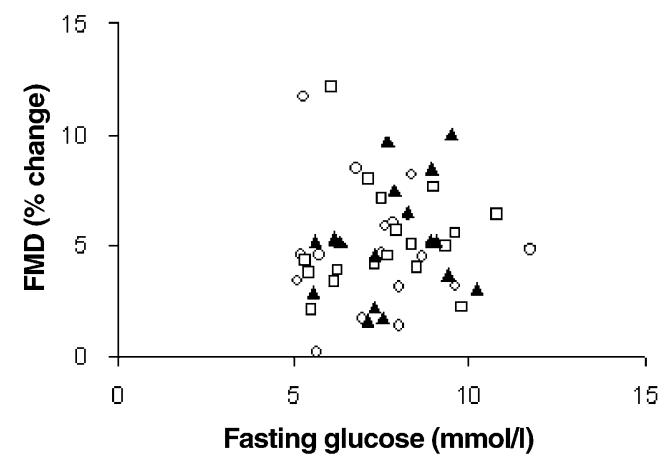

Fig. 1. Fasting glucose concentrations and brachial artery FMD values in individuals with Type 2 diabetes $(n=18$, three visits per subject). Open circles, visit one; open squares, visit two; filled triangles, visit three

ity was similar to that reported by Herrington et al. [12].

The primary measure of variability was the mean difference between three pairs of measurements, expressed as an absolute value (Table 3). Variability in FMD was positively correlated with variability in glucose $(r=0.52, p=0.03)$, insulin $(r=0.47, p=0.05)$ and heart rate $(r=0.48, p=0.04)$, despite the fact that FMD was not related to absolute levels of these parameters. In multivariate analysis, only mean variability in glucose remained an independent predictor, accounting for $35 \%$ of the variance in FMD variability. FMD variability was unrelated to fasting glucose or any of the other health-related variables examined $\left(\mathrm{HbA}_{1} \mathrm{c}\right.$, age, BMI, BP and lipids). Results were unchanged when adjusted for the number of days between visits.

Effects of peak selection on the stability of FMD. As expected, FMD scores were higher when a single frame was used to calculate the peak diameter (Table 5). However, the SD of the repetitions remained stable irrespective of the number of adjacent frames used to define the peak. The lowest CV (indicating higher reliability) was observed when the mean diameter of a single frame was used. 
Table 4. Test-retest reliability of blood flow and blood-flow velocity measurements

\begin{tabular}{lcccc}
\hline & $\begin{array}{l}\text { Resting blood flow } \\
(\mathrm{ml} / \mathrm{min})\end{array}$ & $\begin{array}{l}\text { Peak hyperaemic flow } \\
(\mathrm{ml} / \mathrm{min})\end{array}$ & $\begin{array}{l}\text { Resting flow velocity } \\
(\mathrm{cm} / \mathrm{s})\end{array}$ & $\begin{array}{l}\text { Peak hyperaemic } \\
\text { flow velocity }(\mathrm{cm} / \mathrm{s})\end{array}$ \\
\hline Mean $\pm \mathrm{SD}$ & $109.4 \pm 31.9$ & $740.2 \pm 272.5$ & $0.96 \pm 0.22$ & $1.75 \pm 0.43$ \\
$\mathrm{CV}(\%) \mathrm{a}$ & 17.7 & 21.9 & 10.8 & $0.09 \pm 0.13$ \\
Variability $\left|\mathrm{t}_{1}-\mathrm{t}_{2}\right|$ & $17.4 \pm 13.3$ & $167.4 \pm 105.2$ & $0.14 \pm 0.10$ & $0.37 \pm 0.23$ \\
Variability $\left|\mathrm{t}_{2}-\mathrm{t}_{3}\right|$ & $28.2 \pm 20.0$ & $230.7 \pm 143.8$ & $0.16 \pm 0.14$ & $0.45 \pm 0.31$ \\
Variability $\left|\mathrm{t}_{1}-\mathrm{t}_{3}\right|$ & $26.7 \pm 18.1$ & $196.2 \pm 156.6$ & $0.13 \pm 0.08$ & $0.37 \pm 0.20$ \\
Mean variability & $24.1 \pm 10.9$ & $193.4 \pm 98.1$ & 0.75 & 0.72 \\
$r$ value, $\mathrm{t}_{1} \mathrm{vs}_{2}$ & 0.81 & 0.78 & 0.82 & 0.73 \\
$r$ value, $\mathrm{t}_{2} \mathrm{vs} \mathrm{t}_{3}$ & 0.53 & 0.67 & 0.69 & 0.40 \\
$r$ value $\mathrm{t}_{1} \mathrm{vs} \mathrm{t}_{3}$ & 0.59 & 0.68 & & \\
\hline
\end{tabular}

${ }^{\mathrm{a}} \mathrm{CV}=(100 \times \mathrm{SD}) /$ mean

Table 5. Effect of increasing the number of frames used to calculate the peak diameter after cuff deflation on the stability of FMD estimates

\begin{tabular}{|c|c|c|c|c|}
\hline & Mean FMD (\%) & $\mathrm{SD}^{\mathrm{a}}$ & Mean CV $(\%)^{\mathrm{b}}$ & Mean CV' $(\%)^{\mathrm{C}}$ \\
\hline Peak only & 5.89 & 1.30 & 23.9 & 1.23 \\
\hline Peak \pm 1 & 5.42 & 1.30 & 27.8 & 1.23 \\
\hline Peak \pm 3 & 5.27 & 1.31 & 28.7 & 1.24 \\
\hline Peak \pm 4 & 5.21 & 1.32 & 29.8 & 1.26 \\
\hline Peak \pm 5 & 5.18 & 1.32 & 29.9 & 1.25 \\
\hline
\end{tabular}

a Group average for the SD of the three repeated measurements; ${ }^{b} \mathrm{CV}=(100 \times \mathrm{SD}) / \mathrm{mean} ;{ }^{\mathrm{c}} \mathrm{CV}{ }^{\prime}=(100 \times \mathrm{SD}) /(\mathrm{mean}+100)$

Table 6. Sample sizes required to determine significant effects on FMD in crossover and parallel-arm studies

\begin{tabular}{|c|c|c|c|c|}
\hline \multirow[t]{3}{*}{$\begin{array}{l}\text { Magnitude of the } \\
\text { treatment effect }(\%)\end{array}$} & \multicolumn{2}{|c|}{$\begin{array}{l}\text { Number of subjects in crossover } \\
\text { study when comparing end values }\end{array}$} & \multicolumn{2}{|c|}{$\begin{array}{l}\text { Number of subjects per group when } \\
\text { comparing responses }^{b}\end{array}$} \\
\hline & \multicolumn{2}{|c|}{ Power } & \multicolumn{2}{|c|}{ Power } \\
\hline & 0.80 & 0.90 & 0.80 & 0.90 \\
\hline 5 & 522 & 698 & 1188 & 1589 \\
\hline 20 & 35 & 46 & 76 & 101 \\
\hline 25 & 23 & 30 & 49 & 65 \\
\hline 30 & 17 & 22 & 35 & 46 \\
\hline 35 & 13 & 17 & 26 & 34 \\
\hline 40 & 11 & 14 & 20 & 27 \\
\hline
\end{tabular}

${ }^{\text {a }}$ Calculated using mean $=5.18$ and $\mathrm{SD}=\sqrt{ } 2 \times \mathrm{SD}_{\text {within }}=2.12 ;{ }^{\mathrm{b}}$ calculated using mean $\pm \mathrm{SD}=5.18 \pm 2.26$

Sample size. Table 6 shows sample size requirements for crossover and parallel-arm designs when the magnitude of the treatment effect on FMD can be estimated. If an intervention is expected to increase fasting FMD by $25 \%$ (power $=0.90$ ), 30 subjects would be needed to find significance in a crossover design, while a parallel-arm design would require 65 subjects.

\section{Discussion}

Experts in the measurement of FMD suggest that individual laboratories document test-retest reliability of their measurement protocols by repeatedly testing the same individuals in the absence of changes in medication status, diet or exercise regimen. We have shown that FMD can be reliably measured in healthy individuals with diabetes, and that fasting glucose and insulin concentrations are not correlated with FMD scores measured simultaneously. In the present study, fasting glucose varied by more than $5.56 \mathrm{mmol} / \mathrm{l}$ between individuals and by up to $2.78 \mathrm{mmol} / \mathrm{l}$ within individuals. Thus, we cannot comment on whether or not glucose variations over a broader range would impact FMD scores. Our estimates of test-retest reliability in patients with Type 2 diabetes are in keeping with those 
in samples of healthy adults [12], and our estimates of the CV for FMD are considerably lower than in several other studies.

The variability estimates reported here may be considered conservative given the controlled conditions under which the measurements were collected. Participants were instructed to avoid changes in exercise, diet or medication regimen during the study. Before each test, subjects fasted for $12 \mathrm{~h}$ (confirmed by glucose measurement). Ultrasound studies were conducted after a 45-min habituation period and $20 \mathrm{~min}$ of quiet rest. A single sonographer performed all FMD assessments, and arterial diameters were measured using automated scoring software that had been validated for this purpose [15]. Our design controlled for diurnal variability in FMD [21] and hormone fluctuations caused by the menstrual cycle [22], and there were only minimal changes in heart rate and $\mathrm{BP}$ over the three visits.

Nevertheless, the reported CVs for FMD (in this study, and in many others) are higher than those conventionally accepted for biochemical assays. As noted previously, variability in FMD is likely to result from the combined effects of measurement error, real biological variability and subtle differences in the application of the technique over repeated testing sessions [20]. We found that subjects with more variable values for glucose, insulin and heart rate also showed the greatest variation in FMD across testing days. Variability in glucose was the only independent predictor of variability in FMD.

These results suggest that true biological variability is a significant source of variation in FMD scores. However, the lack of a correlation between fasting glucose concentrations and fasting FMD scores indicates that the relationship between glucose and FMD is complex. It is possible that larger day-to-day fluctuations in glucose are a marker of more advanced disease, and that FMD variability may be even greater in individuals with poorly controlled diabetes. In as far as heart rate, glucose and insulin levels can be controlled by careful subject selection and instruction, researchers are advised to account for these variables in their study design and analyses.

In this study, measurements of hyperaemic flow were also highly variable from one testing session to the next. However, neither FMD nor the volume of blood flow during reactive hyperaemia were significantly altered with repeated testing. Our results showed an unexpected decrease in peak flow velocity during hyperaemia over time. These results highlight the importance of careful study design, including counterbalanced presentation of treatments in crossover studies.

There appears to be no additional benefit (in terms of lower variability) from including diameters from multiple adjacent frames in the calculation of FMD scores. In fact, CV estimates actually increased when more diameters were used to determine peak diameter. The use of automated edge-detection software in this study meant that the value for average diameter within each frame was actually based on dozens of measurements across the segment of vessel in the region of interest. This method substantially reduces the influence of a single aberrant diameter on FMD estimates, and FMD estimates determined using this software may be more reliable than those obtained by traditional methods for measuring arterial boundaries [15].

Although nutritional interventions have been shown to improve FMD in smokers and subjects with elevated cholesterol [13], relatively few of these studies have included patients with diabetes. Using the same study population, we recently reported that individuals with both diabetes and hypertriglyceridaemia showed substantial FMD improvements $4 \mathrm{~h}$ after a meal containing omega-3 fatty acids [14]. The success of future intervention studies is dependent on accurate estimates of within-subject variability and control of extraneous variables that are known to influence FMD. In this study, we have demonstrated that FMD can be measured as reproducibly in patients with diabetes as in persons without diabetes, even in the presence of glucose and insulin fluctuations across testing days.

Acknowledgements. Primary funding was provided by Ross Products Division, Abbott Laboratories (Columbus, Ohio, USA); however, the results were not influenced by Abbott Laboratories. Additional funding was provided by an Albert and Lorraine Kligman Graduate Fellowship awarded to K. D. Hecker. The services provided by the General Clinical Research Center of The Pennsylvania State University are appreciated (NIH grant M01 RR10732). We appreciate technical assistance provided by M. Drum of Wake Forest University School of Medicine, Section of Cardiology, and D. Van Creveld of Acuson (Siemens Ultrasound Division).

\section{References}

1. Schachinger V, Britten MB, Zeiher AM (2000) Prognostic impact of coronary vasodilator dysfunction on adverse long-term outcome of coronary heart disease. Circulation 101:1899-1906

2. Makimattila S, Yki-Jarvinen H (2002) Endothelial dysfunction in human diabetes. Curr Diab Rep 2:26-36

3. Wilson PW, Kannel WB (2002) Obesity, diabetes, and risk of cardiovascular disease in the elderly. Am J Geriatr Cardiol 11:119-123,125

4. Watts GF, Playford DA, Croft KD, Ward NC, Mori TA, Burke V (2002) Coenzyme Q(10) improves endothelial dysfunction of the brachial artery in Type II diabetes mellitus. Diabetologia 45:420-426

5. Mather KJ, Verma S, Anderson TJ (2001) Improved endothelial function with metformin in type 2 diabetes mellitus. J Am Coll Cardiol 37:1344-1350

6. Celermajer DS, Sorensen KE, Bull C, Robinson J, Deanfield JE (1994) Endothelium-dependent dilation in the systemic arteries of asymptomatic subjects relates to coronary risk factors and their interaction. J Am Coll Cardiol 24:1468-1474 
7. Corretti MC, Anderson TJ, Benjamin EJ et al. (2002) Guidelines for the ultrasound assessment of endothelial-dependent flow-mediated vasodilation of the brachial artery: a report of the International Brachial Artery Reactivity Task Force. J Am Coll Cardiol 39:257-265

8. Sinoway LI, Hendrickson C, Davidson WR Jr, Prophet S, Zelis R (1989) Characteristics of flow-mediated brachial artery vasodilation in human subjects. Circ Res 64:32-42

9. Kuvin JT, Karas RH (2003) Clinical utility of endothelial function testing: ready for prime time? Circulation 107: 3243-3247

10. Zizek B, Poredos P (2001) Insulin resistance adds to endothelial dysfunction in hypertensive patients and in normotensive offspring of subjects with essential hypertension. J Intern Med 249:189-197

11. Brook RD, Bard RL, Rubenfire M, Ridker PM, Rajagopalan S (2001) Usefulness of visceral obesity (waist/hip ratio) in predicting vascular endothelial function in healthy overweight adults. Am J Cardiol 88:1264-1269

12. Herrington DM, Fan L, Drum M et al. (2001) Brachial flow-mediated vasodilator responses in population-based research: methods, reproducibility and effects of age, gender and baseline diameter. J Cardiovasc Risk 8:319-328

13. West SG (2001) Effect of diet on vascular reactivity: an emerging marker for vascular risk. Curr Atheroscler Rep 3:446-455

14. West SG, Hecker KD, Mustad VA et al. (2004) Acute effects of monounsaturated fatty acids with and without omega-3 fatty acids on vascular reactivity in individuals with Type-2 diabetes. Diabetologia (in press)

15. Sonka M, Liang W, Lauer RM (2002) Automated analysis of brachial ultrasound image sequences: early detection of cardiovascular disease via surrogates of endothelial function. IEEE Trans Med Imaging 21:1271-1279

16. Mannion TC, Vita JA, Keaney JF Jr, Benjamin EJ, Hunter L, Polak JF (1998) Non-invasive assessment of brachial artery endothelial vasomotor function: The effect of cuff position on level of discomfort and vasomotor responses. Vasc Med 3:263-267

17. Bonora E, Formentini G, Calcaterra F et al. (2002) HOMAestimated insulin resistance is an independent predictor of cardiovascular disease in type 2 diabetic subjects: prospective data from the Verona Diabetes Complications Study. Diabetes Care 25:1135-1141

18. Katz A, Nambi SS, Mather K et al. (2000) Quantitative insulin sensitivity check index: a simple, accurate method for assessing insulin sensitivity in humans. J Clin Endocrinol Metab 85:2402-2410

19. West SG, Hinderliter AL, Wells EC, Girdler SS, Light KC (2001) Transdermal estrogen reduces vascular resistance and serum cholesterol in postmenopausal women. Am J Obstet Gynecol 184:926-933
20. de Roos NM, Bots ML, Schouten EG, Katan MB (2003) Within-subject variability of flow-mediated vasodilation of the brachial artery in healthy men and women: implications for experimental studies. Ultrasound Med Biol 29:401406

21. Etsuda H, Takase B, Uehata A et al. (1999) Morning attenuation of endothelium-dependent, flow-mediated dilation in healthy young men: possible connection to morning peak of cardiac events? Clin Cardiol 22:417-421

22. Hashimoto M, Akishita M, Eto M et al. (1995) Modulation of endothelium-dependent, flow-mediated dilatation of the brachial artery by sex and menstrual cycle. Circulation 92:3431-3435

23. de Roos NM, Siebelink E, Bots ML, Tol A van, Schouten EG, Katan MB (2002) Trans monounsaturated fatty acids and saturated fatty acids have similar effects on postprandial flow-mediated vasodilation. Eur J Clin Nutr 56:674-679

24. de Roos NM, Bots ML, Siebelink E, Schouten E, Katan MB (2001) Flow-mediated vasodilation is not impaired when HDL-cholesterol is lowered by substituting carbohydrates for monounsaturated fat. Br J Nutr 86:181-188

25. de Roos NM, Bots ML, Katan MB (2001) Replacement of dietary saturated fatty acids by trans fatty acids lowers serum HDL cholesterol and impairs endothelial function in healthy men and women. Arterioscler Thromb Vasc Biol 21:1233-1237

26. Liang YL, Teede H, Kotsopoulos D et al. (1998) Non-invasive measurements of arterial structure and function: repeatability, interrelationships and trial sample size. Clin Sci (Lond) 95:669-679

27. Sorensen KE, Celermajer DS, Spiegelhalter DJ et al. (1995) Non-invasive measurement of human endothelium dependent arterial responses: accuracy and reproducibility. Br Heart J 74:247-253

28. Hale G, Paul-Labrador M, Dwyer JH, Merz CN (2002) Isoflavone supplementation and endothelial function in menopausal women. Clin Endocrinol (Oxf) 56:693-701

29. Berry KL, Skyrme-Jones RA, Meredith IT (2000) Occlusion cuff position is an important determinant of the time course and magnitude of human brachial artery flow-mediated dilation. Clin Sci (Lond) 99:261-267

30. Kanani PM, Sinkey CA, Browning RL, Allaman M, Knapp HR, Haynes WG (1999) Role of oxidant stress in endothelial dysfunction produced by experimental hyperhomocyst(e)inemia in humans. Circulation 100:1161-1168

31. Lind L, Hall J, Johansson K (2002) Evaluation of four different methods to measure endothelium-dependent vasodilation in the human peripheral circulation. Clin Sci (Lond) 102:561-567

32. Uehata A, Lieberman EH, Gerhard MD et al. (1997) Noninvasive assessment of endothelium-dependent flow-mediated dilation of the brachial artery. Vasc Med 2:87-92 